\title{
Blind Saccades: An Asynchrony between Seeing and Looking
}

\author{
Claudio de'Sperati ${ }^{1}$ and Gabriel Baud-Bovy ${ }^{2}$ \\ ${ }^{1}$ Visuomotor Functions Laboratory and ${ }^{2}$ Laboratory of Action, Perception, and Cognition, University Vita-Salute San Raffaele, 20132 Milan, Italy
}

Saccades may not always wait for the completion of the perceptual analysis. By taking advantage of a motion-induced illusion of position and of the spontaneous scatter of saccade latency, we showed that in normal observers, regular saccades (latency, $\sim 200 \mathrm{~ms}$ ) were accurately directed to the target, whereas at higher latencies, saccades were increasingly biased by visual motion until they reflected the perceptual illusion. We reconstructed the time course of saccadic direction coding and identified an early phase in which saccades are mostly predictive (latencies less than $\sim 100 \mathrm{~ms}$ ), followed by a phase in which saccades are guided by the target position signal (latencies $\sim 100-250 \mathrm{~ms})$, and a later phase associated with the buildup of mislocalization $(\sim 250-450 \mathrm{~ms})$. This transient dissociation between action and perception indicates that seeing and looking are based on asynchronous processes, possibly because of independent thresholds for saccades and perceptual localization. The metrics of a saccade would then reflect the evolution of cortical visual signals from a predictive state to a perceptual state, passing through an intermediate visuomotor state. If saccades occur during the visuomotor state, they escape the tricks of perception.

Key words: saccades; time; decision process; motion-position; action-perception; visual illusions

\section{Introduction}

A main tenet of an influential theory of visual processing is that visuomotor responses can be at least partly independent of visual perception. According to Goodale and Milner (1992), this duplex nature of vision depends on the emergence of distinct pathways in the primate cerebral cortex, a dorsal, "vision-for-action" stream, and a ventral, "vision-for-perception" stream. Although this theory was originally based on neuropsychological evidence, empirical support has also come from studies of normal observers, in which stimuli that fool perception (illusions) do not always fool action (Goodale and Westwood, 2004). However, several investigators have recently argued that the purported differences between vision-for-action and vision-for-perception in normal observers depend on implicit task demands or on the measuring methods, rather than on genuine dissociations between the two visual channels (Franz, 2001; Kerzel and Gegenfurtner, 2005). Thus, whether or not visually guided movements are always guided by what we see is an open question.

One situation in which a dissociation between action and perception might occur is where a (correct) movement is generated before the illusory percept is formed. In fact, perceptual awareness is a dynamic, time-consuming event, and sometimes perceptual delays can reach 200-300 ms or more (Castiello et al., 1991; Gold and Shadlen, 2000; Sergent et al., 2005) (see also Libet, 2004). In contrast, many visuomotor responses should be both fast and accurate, particularly in life-and-death situations, in

Received 0ct. 17, 2007; accepted March 17, 2008.

This work was partly supported by Ministero dell'Istruzione, Università e Ricerca Grants PRIN-2005057573_003 (C.d.'S.) and 2005095039_003 (G.B.B.). We are grateful to Giorgio Grimoldi for his precious help in data acquisition and computer programming.

Correspondence should be addressed to Claudio de'Sperati, Visuomotor Functions Laboratory, Università VitaSalute San Raffaele, via Olgettina 58, 20132 Milan, Italy. E-mail: desperati.claudio@hsr.it.

D01:10.1523/JNEUROSCI.0352-08.2008

Copyright $\odot 2008$ Society for Neuroscience $\quad$ 0270-6474/08/284317-05\$15.00/0 which an important delay could be fatal. Indeed, visuomotor systems, especially saccades, can react very quickly to a visual event (Prablanc and Jeannerod, 1975; Deubel et al., 1982; Fischer and Ramsperger, 1984; Whitney et al., 2003; Kirchner and Thorpe, 2006). Presumably, such responses cannot wait for the completion of the perceptual analysis (Bridgeman et al., 1997; Hu and Goodale, 2000; Eggert et al., 2002; Blohm et al., 2005) (but see Dassonville and Bala, 2004). However, when, in the few hundreds of milliseconds after stimulus presentation, the visual input turns into a saccade or a percept is not known. Here we reconstructed the transition from stimulus-based to perceptually based saccadic direction coding in the subsecond range by relying on the spontaneous fluctuation of saccadic latency and by exploiting the illusory mislocalization of a flash induced by visual motion (Whitney and Cavanagh, 2000; Durant and Johnston, 2004).

\section{Materials and Methods}

Participants. Six participants ( 3 males and 3 females; age, 22-44) participated in the study. All had normal or corrected-to-normal vision, and five were naive as to the purpose of the study. Informed consent was obtained before the beginning of the experiments, in accordance with the guidelines of the local ethical committee.

Stimuli and tasks. Participants seated $62 \mathrm{~cm}$ in front of a computer screen (Trinitron; Sony, Tokyo, Japan; frame rate, $75 \mathrm{~Hz}$; resolution, $1600 \times 1200$ pixels; background luminance, $\left.26 \mathrm{~cd} / \mathrm{m}^{2}\right)$ in a moderately darkened room. A trial started with the presentation of a central fixation cross. After $1 \mathrm{~s}$, a gray circular arc (thickness, $0.2^{\circ}$ of visual angle; luminance, $65 \mathrm{~cd} / \mathrm{m}^{2}$ ) extending over an angle of $30^{\circ}$ (corresponding to a visual angle of $2.42^{\circ}$ ) was presented in the right or left visual field at 3 or at 9 o'clock, at $5^{\circ}$ of eccentricity (Fig. 1). After a randomized interval (450-2150 ms, aging foreperiod) the arc started revolving at constant angular speed $(200 \%, 0.555 \mathrm{~Hz})$ in the clockwise or counterclockwise direction. Both the side of presentation and the motion direction were randomly interleaved. A white spot (diameter, $0.4^{\circ}$; luminance, $125 \mathrm{~cd} /$ $\mathrm{m}^{2}$; eccentricity, $5.3^{\circ}$ ) was displayed for one video frame when the arc started moving. Flash occurrence was measured with a photocell. The 


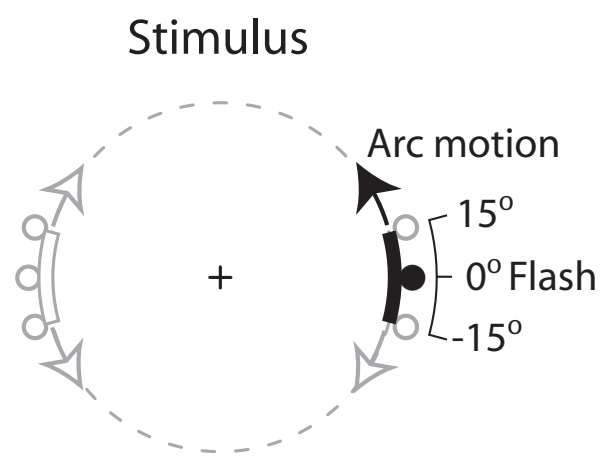

Motion \& Gap Tasks
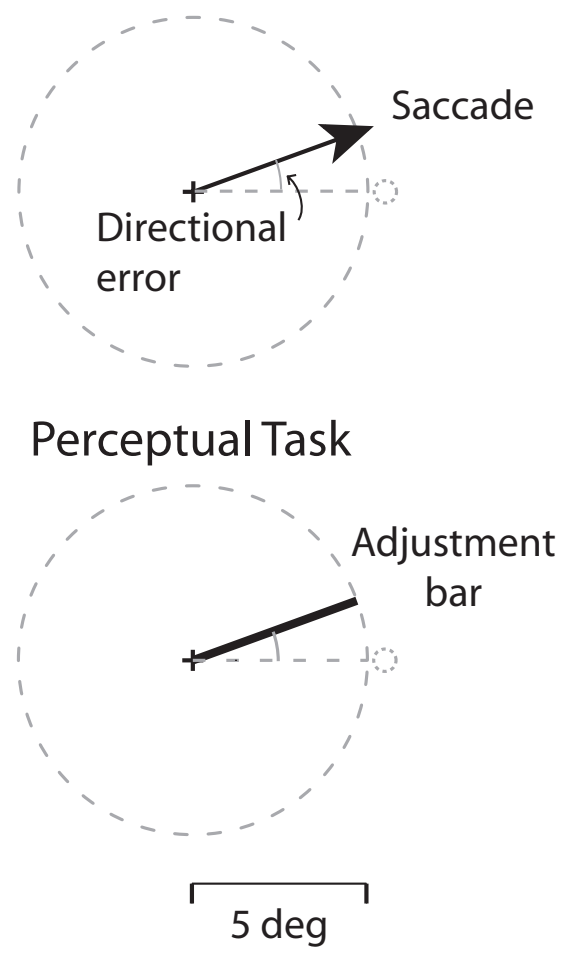

Figure 1. Stimulus and tasks. The arcstarted moving simultaneously with the flash, to which participants had to orient a pointer (perceptual task) or make a saccade (motion and gap tasks). The three flash positions (displacement, $+15,0$, and $-15^{\circ}$ ), the two motion directions (clockwise and counterclockwise), and the two stimulus presentation sides (right and left) are illustrated (the elements in black depict a prototypical trial). The angle between response direction (thick line or arrow) and flash direction (dashed line) was the directional error. For graphical purposes, the layout of the figure is somewhat arbitrary.

flash was randomly presented at the trailing edge of the arc (displaced backward by an angle of $15^{\circ}$, defined as " $-15^{\circ}$ " displacement), centered (" $0^{\circ}$ "), or at the leading edge (" $+15^{\circ}$ ”). The arc moved for 2.25 or $3.15 \mathrm{~s}$ before disappearing.

In the perceptual task, participants viewed the stimuli while fixating the central position and located the flash position by rotating a pointer line with keyboard keys. The directional error between the flash angle and the pointer angle was taken (positive value $=$ error in the motion direction). In the main task (motion task), participants made a saccade to the flash, starting from central fixation (overlap paradigm). No constraints for urgency or accuracy were given, and participants received no feedback. Urgency was instead required in the gap task, identical to the motion task except that participants were urged to make the saccade as early as possible. To further promote short-latency saccades, the fixation cross was extinguished $200 \mathrm{~ms}$ before the flash (gap paradigm). The first control task (no-motion task) was identical to the motion task except that
Table 1. Mean $\pm S D$ of directional error and saccadic latency in the five tasks

\begin{tabular}{lcl}
\hline & Directional error $\left(^{\circ}\right)$ & Saccadic latency $(\mathrm{ms})$ \\
\hline Perceptual task & $10.14 \pm 10.52$ & \\
Motion task & $4.13 \pm 8.05$ & $320 \pm 78$ \\
Gap task & $3.47 \pm 8.08$ & $221 \pm 65$ \\
No motion task & $0.07 \pm 6.04$ & $337 \pm 63$ \\
Stationary arc task & $0.08 \pm 8.21$ & $355 \pm 108$ \\
\hline
\end{tabular}

there was no arc whatsoever. The second control task (stationary arc task) allowed us to ascertain whether or not the mere presence of two objects (the arc and the flash) in the visual display influenced saccadic localization: the arc was displayed simultaneously with the flash for one video frame, displaced on the circular trajectory by an angle between 45 and $62^{\circ}$ in the clockwise or counterclockwise direction, relative to the horizontal midline (see "Angular displacement" in the supplemental materials, available at www.jneurosci.org).

The five tasks were administered in the following order: motion task, no-motion task, stationary arc task, gap task, and perceptual task, with one (no-motion task, stationary arc task, and perceptual task) or two (motion task and gap task) experimental blocks. A block comprised 60 trials $(2$ sides $\times 2$ motion directions $\times 3$ flash displacements $\times 5$ repetitions).

Eye movement recording. Two-dimensional eye movements were recorded through infrared oculometry (Dr. Bouis; precision, $<0.3^{\circ}$; sampling rate, $1000 \mathrm{~Hz}$; resolution, 16 bit) in head-restrained conditions. The traces were low-pass filtered $(150 \mathrm{~Hz})$ and calibrated. Saccade onset and offset were identified when instantaneous tangential velocity exceeded $20 \%$ and dropped below $30 \%$, respectively. Only primary saccades with amplitudes between 2 and $8^{\circ}$ were considered. We excluded saccades whose direction departed $>60^{\circ}$ from the flash direction, as well as trials containing ocular artifacts.

Data analyses. We performed separate repeated-measures ANOVAs of the directional error for each task and used univariate ANOVA with participants as random factor to assess the effect of latency, because some latency bins were empty in some participants. These analyses were double checked with mixed-models ANOVAs, which gave the same results (data not shown). Post hoc Tukey's honestly significant difference and one-sample Student's $t$ tests were also used. The two-component model was fitted with the Golub-Pereyra algorithm for partially linear leastsquares models. In the text, values are reported as mean $\pm \mathrm{SD}$.

\section{Results}

In the perceptual task, the flash was perceived shifted in the direction of visual motion relative to its physical position (mean directional error, $10.1 \pm 10.5^{\circ}$, corresponding to $0.81^{\circ}$ of visual angle; $p<0.001)$. The error was independent of the side of flash presentation $(p=0.417)$ and the direction of motion $(p=$ $0.790)$, but their interaction was significant $(p=0.004)$, indicating a larger error when the arc moved upward. Subjectively, the flash appeared motionless.

In the motion task, participants made saccades to the flash with a mean latency of $320 \pm 78 \mathrm{~ms}$ (Table 1). These relatively long latencies, compared with latencies usually reported for visually guided saccades $(\sim 200 \mathrm{~ms})$, could be caused by the transient nature of the target, the uncertainty about its position, the overlap paradigm, the fact that participants were not urged to respond, or the relatively long foreperiod. The end points of saccades were biased in the direction of motion by $4.13 \pm 8.05^{\circ}$, regardless the side of presentation $(p=0.248)$ and the motion direction $(p=0.873)$. The interaction was significant $(p=$ 0.035 ). The directional error was smaller than in the perceptual task $(p<0.001)$, but larger than in the no-motion or stationary arc task $(p<0.001)$, in which there was no error. Thus, the directional error was specifically introduced by visual motion. 


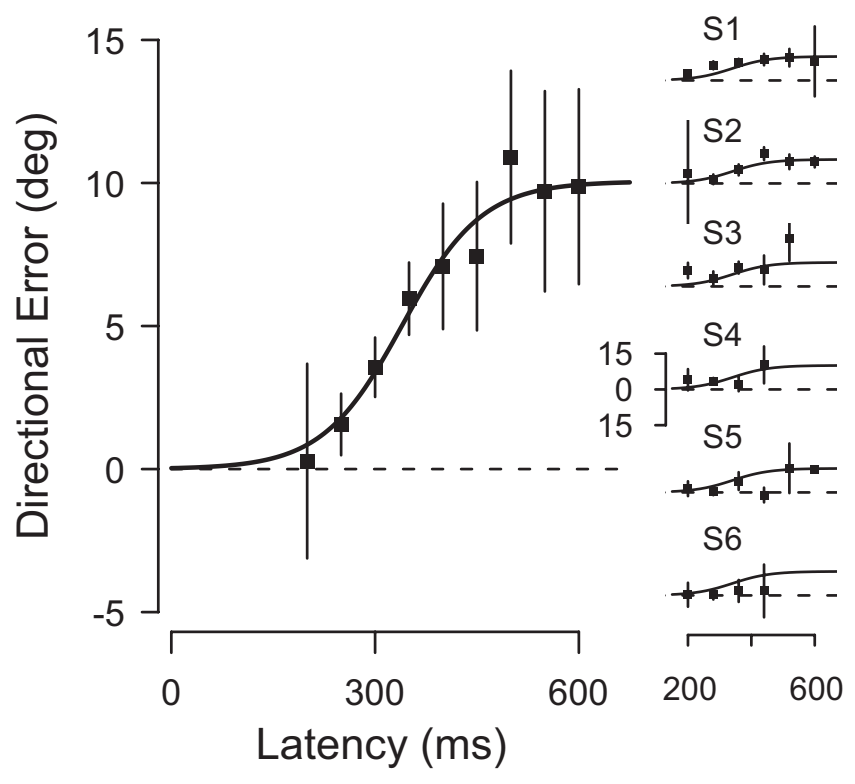

Figure 2. Left, Buildup of the motion-induced saccadic directional error in the motion task, pooled over all subjects, together with the logistic fit. Bin width, $50 \mathrm{~ms}$. Error bars represent $95 \%$ confidence interval. Right, Individual plots. The logistic fit obtained from the pooled data is plotted for comparison. Bin width, 80 ms. Error bars represent $80 \%$ confidence interval. S1-S6, Subjects 1-6.

Note that the latency range of the saccades in the no-motion and stationary arc tasks was similar to that of the motion task.

At first sight, then, it would appear that not only perceptual judgments, but also saccades, were subjected to the motioninduced mislocalization illusion, although to a lesser extent. However, by plotting the saccadic directional error against saccadic latency, a quite different picture emerged: the mean directional error increased gradually as latency increased ( $p=0.016)$, passing from $0.28 \pm 8.24^{\circ}$ at $200 \mathrm{~ms}$ (first bin) (Fig. 2, left) to $9.87 \pm 3.25^{\circ}$ at $600 \mathrm{~ms}$ (last bin). Saccadic precision remained constant across latency bins (nonsignificant main effect of latency; $p=0.998$ ), and in a normal range [mean SD of directional saccadic error, $7.02 \pm 3.09^{\circ}$, corresponding to $0.57 \pm 0.25^{\circ}$ of visual angle (de'Sperati and Deubel, 2006)], which indicates that saccadic accuracy did not depend on the uncertainty of the response.

We checked that short-latency saccades in the motion task were truly directed to the target and not simply released as a "default," anticipatory response toward the midposition of the arc. The error was $-1.59 \pm 8.12^{\circ}$ for saccades with latencies $\leq 250$ $\mathrm{ms}$ directed to $+15^{\circ}$ flash position, and $1.24 \pm 8.02^{\circ}$ for the $0^{\circ}$ flash displacement, which was not significantly different from zero in either case $(p>0.2)$. For the $-15^{\circ}$ flash displacement, the error was $6.28 \pm 7.13^{\circ}$, indicating that these saccades were on average directed halfway in between the target and the midposition of the $\operatorname{arc}(p<0.001)$.

The buildup of the directional error was described by a logistic function (accounted variance, $8.4 \%$ across all trials; $p<0.01$ ), which had an asymptotic value of $10.1^{\circ}$. The logistic function was chosen for its simple asymptotic properties, but this choice is somewhat arbitrary. The buildup of mislocalization was present also in individual participants (Fig. 2, right). Because of the smaller and more irregular samples, especially at higher latencies, the fit was not performed for the individual participants. However, the individual time courses of the directional error matched the fit performed on the pooled data (thin lines). The buildup of the error was most clearly visible in those participants who produced most of the long-latency saccades (saccades with latencies $>400 \mathrm{~ms}$ formed $>26 \%$ of all saccades for participants 1 and 2, $\sim 12 \%$ for participants 3 and 5 , and only $\sim 3 \%$ for participants 4 and 6), which further corroborates the idea that the saccadic directional error takes time to emerge.

The events immediately after the flash presentation could be analyzed in the gap task, in which saccade latencies almost never exceeded $350 \mathrm{~ms}(221 \pm 65 \mathrm{~ms})$. Very short-latency saccades (<120 ms; "express" saccades) (Fischer and Ramsperger, 1984) exhibited a significant directional error $\left(-11.80 \pm 6.63^{\circ}\right.$ and $13.03 \pm 3.04^{\circ}$ for the 15 and $-15^{\circ}$ flash displacements respectively; $p<0.001$ ), indicating that at these latencies, saccades were directed by default toward the center of the arc. In the range of latency overlap between the two tasks (200-350 ms), the directional error was not significantly different between the motion and gap tasks for all three targets $(p>0.2)$.

We then analyzed the entire time course of saccade direction by pooling together data from the gap and motion tasks (Fig. 3). Saccades with latencies $<50 \mathrm{~ms}$ were purely anticipatory and were exclusively directed toward the center of the arc. Over the following $100 \mathrm{~ms}$, the saccade direction shifted toward the corresponding target. This shift was completed for saccades with $\sim 200$ ms latency with the $+15^{\circ}$ flash displacement, and only partially so for saccades toward the $-15^{\circ}$. Finally, at latencies more than $\sim 250 \mathrm{~ms}$, saccade direction started to shift in the direction of visual motion, until a steady state was reached, at latencies of $\sim 450 \mathrm{~ms}$. We modeled the time course of the saccade direction $\left(y_{i}\right)$ toward each one of the three flash displacements $(i=-15,0$, 15) with a sum of two logistic functions:

$$
y_{i}=\frac{a_{1 i}}{1+e^{-c_{1 i}\left(x-b_{1 i}\right)}}+\frac{a_{2 i}}{1+e^{-c_{2 i}\left(x-b_{2 i}\right)}},
$$

where the variable $x$ is the latency. For the $0^{\circ}$ flash displacement, the model included a constant $\left(a_{1 i}\right)$ in place of the first logistic component. The average residual error over all trials was $7.07^{\circ}$, which was similar to the residual error in individual participants (supplemental Fig. 1, available at www.jneurosci.org as supplemental material). We defined the onset and the time-to-steadystate as the time at which each component of the model exceeded 5 and $95 \%$ of its maximum value, respectively. The estimates of the steady state of the first component are very close to the corresponding flash position $\left(a_{1 i}=-15.1,1.1\right.$, and 14.7 for the -15 , 0 , and $15^{\circ}$ displacements, respectively), in line with the idea that this component indicates the effect of target position on the saccade direction. The estimates of the steady state of the second component were all positive, which suggests that this component reflects the bias of visual motion on saccade programming $\left(a_{2 i}=\right.$ 9.3, 7.9, and 9.5 for the $-15,0$, and $15^{\circ}$ flash displacements, respectively). The onset and steady-state times of the first component were 38 and $137 \mathrm{~ms}$ for the $+15^{\circ}$ flash displacement $\left(c_{11}\right.$ $=0.015)$, and 32 and $421 \mathrm{~ms}$ for the -15 flash displacement $\left(c_{13}\right.$ $=0.010)$. These figures for the onset times probably underestimate their true values, because $\sim 50 \mathrm{~ms}$ is the minimum latency for a visual signal to affect saccades (Deubel et al., 1982). The onset and offset times of the second component were 273 and 431 $\mathrm{ms}$ for the $+15^{\circ}$ flash condition $\left(c_{21}=0.0356\right), 278$ and $532 \mathrm{~ms}$ for the $0^{\circ}$ flash condition $\left(c_{22}=0.0232\right)$, and 235 and $421 \mathrm{~ms}$ the $-15^{\circ}$ flash condition $\left(c_{23}=0.0373\right)$. Note the consistency of most estimates across the three flash conditions. The gradual buildup of the second component could reflect a slow action of motion signal, but it could also arise from the pooling of several 
trials, each with an abrupt transition. In that case, the time window of the second component defines the temporal boundaries for the transition.

\section{Discussion}

The main result of our study is that visual motion affected saccades with latencies above $\sim 250 \mathrm{~ms}$, that is, $\sim 150 \mathrm{~ms}$ after the target position signal started to guide them. This motion-induced bias was similar to the one observed when participants adjusted a bar to the perceived position of the flash, a mode of response that is commonly used to investigate visual illusions. Notice that the flash appeared motionless, indicating that the illusion was stable.

These findings can be explained as an asynchrony between seeing and looking. Both saccades and visual perception are thought to depend on rise-to-threshold decision mechanisms, in which the moment a threshold is reached determines when a saccade occurs or when perception is attained (Gold and Shadlen, 2000; Reddi and Carpenter, 2000; Carpenter, 2004; Gold and Shadlen, 2007; Ploran et al., 2007). The asynchrony may originate from two distinct systems for saccades and perceptual localization, each with its own temporal dynamics of the decision process (Goodale and Westwood, 2004; Sakagami et al., 2006). Another possibility is that the asynchrony derives from independent thresholds for saccades and perceptual localization, which would then pick up the visual signal at different moments of its temporal evolution (the "rise"). [In principle, also the baseline activity, and not only the threshold level, could be independent for saccadic and perceptual decisions. In terms of response times, changing the threshold or the baseline is behaviorally equivalent (Reddi and Carpenter, 2000).] Because we could not measure the buildup of perceptual mislocalization, in principle both hypotheses are plausible. For simplicity, we assume that the sensory mechanisms are not doubled for saccades and visual perception and that the asynchrony depends on independent thresholds. If the fluctuation of the saccadic threshold is large enough, saccades will "sample" the entire process of sensory accumulation. The frontal eye fields, the parietal eye fields, the prefrontal cortex, and/or the basal ganglia may play a pivotal role in triggering a saccade with either a short or a long latency (Findlay and Walker, 1999; Schall and Thompson, 1999; Sakagami et al., 2006).

That the saccadic system can access sensory signals before the perceptual threshold is reached is also suggested by a study in the monkey. Under condition of degraded visual motion that slows down the accumulation of sensory evidence, saccades have been forced to use a still-incomplete motion signal (Gold and Shadlen, 2000, 2007). Although saccades were evoked by microstimulation of the frontal eye fields, the study suggests that also naturally occurring saccades could sometimes access early visual signals (see also Reddi et al., 2003). These early saccades could be functional to visual perception. Together with predictive remapping of visual features (Ross et al., 2001; Melcher, 2007), they can be part of a visual preprocessing phase culminating when an object of interest falls on the fovea, that is, after the saccade. This may explain why sometimes ultrafast saccades can be successfully directed to a complex image (Kirchner and Thorpe, 2006): positional cues for saccades may be effective very early after stimulus presentation, before the completion of the perceptual analysis.

Long-latency saccades would instead occur at a time when perceptual processes are completed. Indeed, perceptual delays in the order of few hundreds of milliseconds are not uncommon (Castiello et al., 1991; Gold and Shadlen, 2000; Sergent et al., 2005) and could depend on the recruitment of high-order areas in the ventral stream (Goodale and Milner, 1992) or of a more widespread cortical network (Dehaene et al., 2006), where motion could also be added (Nishida and Johnston, 1999; Sunaert et al., 1999). Furthermore, there is increasing evidence that timeconsuming cortical feedback loops are crucial to shape the sensory input, ultimately producing a unitary perceptual representation (Lamme and Roelfsema, 2000; Hochstein and Ahissar, 2002; Dehaene et al., 2006; Enns and Di Lollo, 2006). It is tempting to speculate that regular, short-latency saccades are associated with the completion of the forward sweep of cortical visual processing, whereas saccades with longer latencies become progressively influenced by the back-propagating reentrant signals. This may correspond to the passage from an "absolute" metrics, in which the visual context (in our case, the spatial relation between the flash and the moving arc) is not yet fixed, to a "relative" metrics, typical of visual perception ( $\mathrm{Hu}$ and Goodale, 2000; Bruno, 2001; Shi and de'Sperati, 2008). In this dynamic view of saccadic latency, the metrics of a saccade would reflect the state of cortical visual signals at the time the oculomotor command is 
triggered. The temporal scheme derived from our data suggests an evolution from a predictive state to a perceptual state, passing through an intermediate visuomotor state. If the threshold for saccades is reached during the visuomotor state, blind saccades are released, which escape the tricks of perception.

Our study provides a clear example of a transient dissociation between action and perception in normal observers, regardless of whether the perceptual illusion formed shortly after stimulus presentation, when saccades are correctly directed to the target, or with a long delay, in which case perceptual awareness lags saccades. Importantly, the same movement in the same experimental condition and with the same task can either resist or reflect the illusion, depending entirely on latency fluctuations in the subsecond range. This is an important difference from previous studies, and may help understanding why action-perception dissociations can easily go undetected (Kerzel and Gegenfurtner, 2005; Knox, 2006; Eckstein et al., 2007).

\section{References}

Blohm G, Missal M, Lefevre P (2005) Processing of retinal and extraretinal signals for memory-guided saccades during smooth pursuit. J Neurophysiol 93:1510-1522.

Bridgeman B, Peery S, Anand S (1997) Interaction of cognitive and sensorimotor maps of visual space. Percept Psychophys 59:456-469.

Bruno N (2001) When does action resist visual illusions? Trends Cogn Sci 5:379-382.

Carpenter RH (2004) Contrast, probability, and saccadic latency; evidence for independence of detection and decision. Curr Biol 14:1576-1580.

Castiello U, Paulignan Y, Jeannerod M (1991) Temporal dissociation of motor responses and subjective awareness. A study in normal subjects. Brain 114:2639-2655.

Dassonville P, Bala JK (2004) Perception, action, and Roelofs effect: a mere illusion of dissociation. PLoS Biol 2:e364.

Dehaene S, Changeux JP, Naccache L, Sackur J, Sergent C (2006) Conscious, preconscious, and subliminal processing: a testable taxonomy. Trends Cogn Sci 10:204-211.

de'Sperati C, Deubel H (2006) Mental extrapolation of motion modulates responsiveness to visual stimuli. Vision Res 46:2593-2601.

Deubel H, Wolf W, Hauske G (1982) Corrective saccades: effect of shifting the saccade goal. Vision Res 22:353-364.

Durant S, Johnston A (2004) Temporal dependence of local motion induced shifts in perceived position. Vision Res 44:357-366.

Eckstein MP, Beutter BR, Pham BT, Shimozaki SS, Stone LS (2007) Similar neural representations of the target for saccades and perception during search. J Neurosci 27:1266-1270.

Eggert T, Sailer U, Ditterich J, Straube A (2002) Differential effect of a distractor on primary saccades and perceptual localization. Vision Res 42:2969-2984.

Enns JT, Di Lollo V (2006) A reentrant view of visual masking, object substitution, and response priming. In: The first half second (Ogmen $\mathrm{H}$, Breitmeyer B, eds), pp 127-148. Cambridge, London: MIT.

Findlay JM, Walker R (1999) A model of saccade generation based on parallel processing and competitive inhibition. Behav Brain Sci 22:661-722.

Fischer B, Ramsperger E (1984) Human express saccades: extremely short reaction times of goal directed eye movements. Exp Brain Res 57:191-195.
Franz VH (2001) Action does not resist visual illusions. Trends Cogn Sci 5:457-459.

Gold JI, Shadlen MN (2000) Representation of a perceptual decision in developing oculomotor commands. Nature 404:390-394.

Gold JI, Shadlen MN (2007) The neural basis of decision making. Annu Rev Neurosci 30:535-574.

Goodale MA, Milner AD (1992) Separate visual pathways for perception and action. Trends Neurosci 15:20-25.

Goodale MA, Westwood DA (2004) An evolving view of duplex vision: separate but interacting cortical pathways for perception and action. Curr Opin Neurobiol 14:203-211.

Hochstein S, Ahissar M (2002) View from the top: hierarchies and reverse hierarchies in the visual system. Neuron 36:791-804.

Hu Y, Goodale MA (2000) Grasping after a delay shifts size-scaling from absolute to relative metrics. J Cogn Neurosci 12:856-868.

Kerzel D, Gegenfurtner KR (2005) Motion-induced illusory displacement reexamined: differences between perception and action? Exp Brain Res 162:191-201.

Kirchner H, Thorpe SJ (2006) Ultra-rapid object detection with saccadic eye movements: visual processing speed revisited. Vision Res 46:1762-1776.

Knox PC (2006) The effect of Kanizsa's compression illusion on reflexive saccades. Exp Brain Res 175:764-768.

Lamme VA, Roelfsema PR (2000) The distinct modes of vision offered by feedforward and recurrent processing. Trends Neurosci 23:571-579.

Libet B (2004) Mind time. In: The temporal factor in consciousness. Cambridge, MA: Harvard UP.

Melcher D (2007) Predictive remapping of visual features precedes saccadic eye movements. Nat Neurosci 10:903-907.

Nishida S, Johnston A (1999) Influence of motion signals on the perceived position of spatial pattern. Nature 397:610-612.

Ploran EJ, Nelson SM, Velanova K, Donaldson DI, Petersen SE, Wheeler ME (2007) Evidence accumulation and the moment of recognition: dissociating perceptual recognition processes using fMRI. J Neurosci 27:11912-11924.

Prablanc C, Jeannerod M (1975) Corrective saccades: dependence on retinal reafferent signals. Vision Res 15:465-469.

Reddi BA, Carpenter RH (2000) The influence of urgency on decision time. Nat Neurosci 3:827-830.

Reddi BA, Asrress KN, Carpenter RH (2003) Accuracy, information, and response time in a saccadic decision task. J Neurophysiol 90:3538-3546.

Ross J, Morrone MC, Goldberg ME, Burr DC (2001) Changes in visual perception at the time of saccades. Trends Neurosci 24:113-121.

Sakagami M, Pan X, Uttl B (2006) Behavioral inhibition and prefrontal cortex in decision-making. Neural Netw 19:1255-1265.

Schall JD, Thompson KG (1999) Neural selection and control of visually guided eye movements. Annu Rev Neurosci 22:241-259.

Sergent C, Baillet S, Dehaene S (2005) Timing of the brain events underlying access to consciousness during the attentional blink. Nat Neurosci 8:1391-1400.

Shi Z, de'Sperati C (2008) Motion-induced positional biases in the flash-lag configuration. Cogn Neuropsychol, in press.

Sunaert S, Van Hecke P, Marchal G, Orban GA (1999) Motion-responsive regions of the human brain. Exp Brain Res 127:355-370.

Whitney D, Cavanagh P (2000) Motion distorts visual space: shifting the perceived position of remote stationary objects. Nat Neurosci 3:954-959.

Whitney D, Westwood DA, Goodale MA (2003) The influence of visual motion on fast reaching movements to a stationary object. Nature 423 : 869-873. 